\title{
Case Report \\ Late Diagnosis of Silent Thoracic Aortic Rupture Presented as a Right Pleural Effusion
}

\author{
Meletios A. Kanakis, ${ }^{1}$ Vassilios G. Papavassiliou, ${ }^{2}$ Polivios Drosos, ${ }^{1}$ \\ Elias A. Kaperonis, ${ }^{2}$ George Benakis, ${ }^{1}$ and Achilleas G. Lioulias ${ }^{1}$ \\ ${ }^{1}$ Thoracic Surgery Department, Sismanoglio General Hospital, 1 Sismanogliou Street, Marousi, 15126 Athens, Greece \\ ${ }^{2}$ Department of Vascular Surgery, Sismanoglio General Hospital, 1 Sismanogliou Street, Marousi, 15126 Athens, Greece
}

Correspondence should be addressed to Meletios A. Kanakis, meletis_kanakis@yahoo.gr

Received 20 May 2012; Revised 1 December 2012; Accepted 15 December 2012

Academic Editor: T. A. Salerno

Copyright (C) 2012 Meletios A. Kanakis et al. This is an open access article distributed under the Creative Commons Attribution License, which permits unrestricted use, distribution, and reproduction in any medium, provided the original work is properly cited.

Patients with ruptured thoracic aortic aneurysm rarely present in a stable clinical condition. A man was referred to our hospital with the diagnosis of ruptured saccular aneurysm of the descending thoracic aorta. He successfully underwent both endovascular graft repair and open thoracotomy.

\section{Introduction}

Rupture of an aneurysm of the thoracic aorta is an emergency condition, which requires prompt diagnosis and treatment. However, in very rare cases, patients may present in stable clinical condition $[1,2]$. We describe a patient with rupture of a descending thoracic aortic aneurysm into the right pleural cavity with some unusual clinical characteristics, making initial correct diagnosis more difficult.

\section{Case Report}

A 65-year-old male was admitted to a regional hospital complaining of progressive cough and respiratory discomfort. The patient had a disease-free history, but for a cervical abscess and mediastinitis due to neglected dental inflammation, for which he had been submitted to drainage through a right lateral cervical incision two years before. Chest radiography revealed right pleural effusion. Apart from anemia (Ht: 32\%) and elevated CRP, all other parameters were normal. Symptoms recessed after a total of $1,000 \mathrm{~mL}$ nonclotting bloody fluid drainage through thoracentesis. The fluid analysis showed hematocrit $6.2 \%$, WBC $2.4 \times$ 10 cells/L with $22 \%$ neutrophils and $75 \%$ lymphocytes, glucose $84 \mathrm{mg} / \mathrm{dL}$, LDH $832 \mathrm{IU} / \mathrm{L}$, and protein $5.5 \mathrm{~g} / \mathrm{dL}$, and its cytologic examination was negative for malignancy. The differential diagnosis was quite broad, including most lesions producing exudative fluid such as tumors of the lung, pleura, or mediastinum. During the next days the patient was mildly febrile, while cough and respiratory discomfort regressed. Computed tomographic scan of the chest confirmed the right pleural effusion along with atelectasis and revealed a saccular aneurysm of the descending aorta in front of T9 vertebra (Figure 1). It measured $2.4 \mathrm{~cm}, 1.5 \mathrm{~cm}$, and $2.6 \mathrm{~cm}$ in longitudinal, transverse, and oblique diameters, respectively, and communicated with thoracic aorta through a lumen of $16 \times 10 \mathrm{~mm}$ width. The aortic diameter above the orifice was $32 \mathrm{~mm}$ and below the orifice $30 \mathrm{~mm}$. The radiologist assumed that minor rupture might exist. The patient was transferred to a tertiary hospital immediately.

Upon arrival at our department, the patient experienced respiratory stress necessitating chest tube drainage of the right hemithorax a few hours later. At insertion of the chest tube, clots along with nonclotting bloody fluid were noted. Chest radiography showed right hemithorax opacity and atelectasis. The patient presented low evening fever, while blood culture was negative. A combined repair was decided. Under general anesthesia, first, a thoracic endovascular graft (GORETAG thoracic endoprosthesis) was introduced through the right common femoral artery into the descending thoracic aorta at the region of the saccular aneurysm under direct fluoroscopic guidance. The size of the endograft 

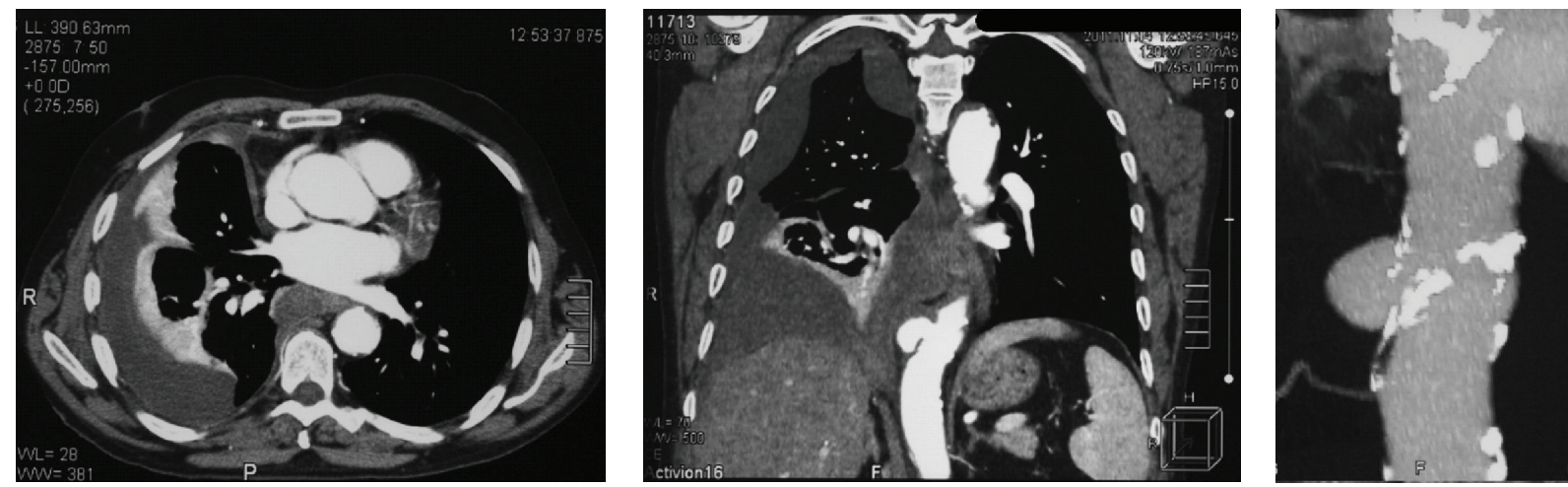

FIGURE 1: Computed tomographic scan of the chest depicting the right pleural effusion along with atelectasis and the saccular aneurysm of the descending aorta.
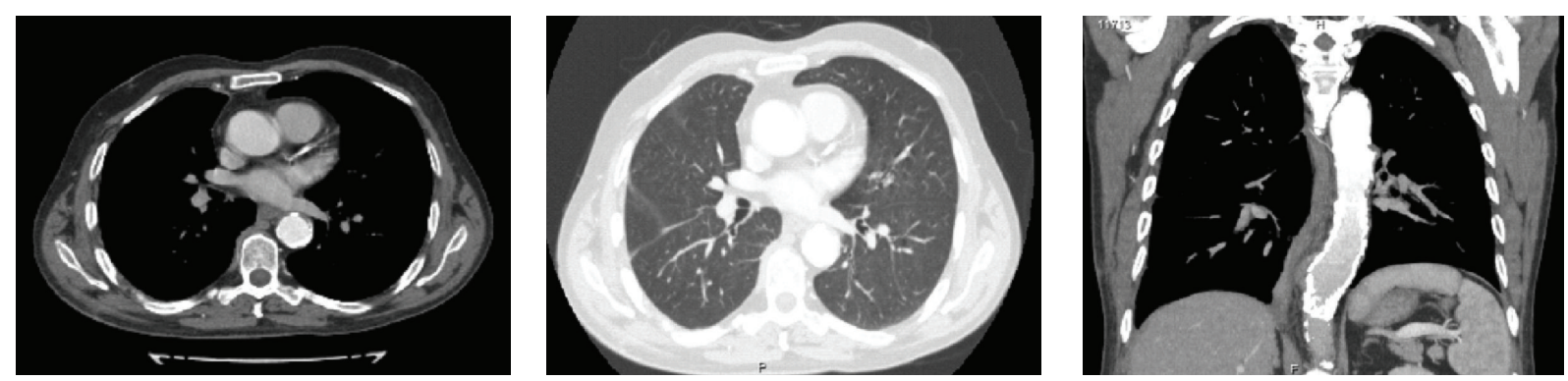

FIGURE 2: A follow-up CT angiography, three months later showed a smooth and patent endograft with no migration or endoleak. The lung is fully re-expanded.

was based on these measurements plus an oversize of around $15 \%$ and enough length to secure safe landing and minimize the risk of endoleak. The device was the straight nontapered GoreTag thoracic endoprosthesis with $37 \mathrm{~mm}$ diameter and $10 \mathrm{~cm}$ length. After deployment of the endograft minimal endoleak was still visible. Ballooning with the trilobe balloon improved wall apposition of the stent graft. Completion angiography showed excellent patency of the endograft without any sign of endoleak. Right after, a right thoracotomy was performed. A large amount of clots were removed. At the region of the saccular aneurysm multiple postinflammatory mediastinal adhesions were recognized. The postoperative course was uneventful, and the patient was discharged from the hospital 8 days after the operation.

A follow-up CT angiography, three months later, showed a smooth and patent endograft with no migration or endoleak (Figure 2). The patient has returned to normal life activity.

\section{Discussion}

Most atherosclerotic aneurysms are found in patients who are older than 60 years. They are asymptomatic and are found as a mediastinal mass during routine examination $[3,4]$. Symptoms and signs are usually related to rapid enlargement, which creates pressure on surrounding tissues and structures.

Aortic dissection or rupture is a major reported cause of hemothorax, which usually presented on left side for anatomical reasons [5]. There are some cases of rightsided hemothorax due to ruptured descending thoracic aneurysm of nontraumatic origin that have been described in the English literature $[1,2,6,7]$. Most of them had an acute presentation and needed urgent surgical management. Baharloo et al. described a patient with a right-sided bleeding due to a fusiform aneurysm of the descending thoracic aorta ruptured along the right side of its wall, causing, however, hemorrhagic shock. The formation of a clot at the site of the tear prevented fatal exsanguinations [2]. In the case of the present patient, hemorrhagic shock was absent, and the patient remained undiagnosed for a period of 10 days. The mediastinal adhesions due to his previous mediastinitis possibly played a major role in hampering continuous bleeding from the ruptured aneurysmal wall.

Once the diagnosis of aortic rupture is established, a multidisciplinary decision for further management with surgery or endovascular stenting should be undertaken. Several factors need to be taken into account, including the patient's risk factors, patient's status (stable or unstable), and institutional factors (availability and resources). Open surgical repair is performed via a left posterolateral thoracotomy with or without cardiopulmonary bypass support and postoperative mortality ranges from $15 \%$ to $30 \%$, and the incidence of paraplegia is reported to be $14 \%$ [8]. Endovascular stent grafting approach has at least equal short-term results in comparison with traditional open surgical repair and can be considered a safe alternative treatment modality in the therapeutic algorithm of thoracic aortic injury $[8,9]$. The right-sided rupture of thoracic aorta was a significant factor for choosing the endovascular stent 
grafting approach. Furthermore, the right thoracotomy was imperative due to the long presence of clots in the thoracic cavity, which impaired lung expansion and would probably lead to empyema and subsequent trapped lung.

The diagnosis of a ruptured thoracic aorta can be misdiagnosed due to its atypical clinical presentation. A thoracic aortic rupture should always be included in differential diagnosis even in a stable patient with right hemorrhagic pleural effusion. Furthermore, the current therapeutic armamentarium offers excellent results by endovascular management of thoracic aorta.

\section{References}

[1] P. Totaro, N. Degno, D. Sprini, and V. Argano, "Unusual presentation of ruptured descending thoracic aortic aneurysm," Journal of Cardiovascular Medicine, vol. 8, no. 4, pp. 291-292, 2007.

[2] F. Baharloo, R. Verhelst, P. Collard, and T. Pieters, "Rupture of aortic aneurysm with right-sided haemothorax," European Respiratory Journal, vol. 13, no. 2, pp. 465-467, 1999.

[3] G. Rábago, A. Martín-Trenor, and J. L. López-Coronado, "Chronic aneurysm of the descending thoracic aorta. Presenting with right pleural effusion and left phrenic paralysis," Texas Heart Institute Journal, vol. 26, no. 1, pp. 96-98, 1999.

[4] R. D. Harris, J. A. Usselman, V. C. Vint, and M. A. Warmath, "Computerized tomographic diagnosis of aneurysms of the thoracic aorta," Computerized Tomography, vol. 3, no. 2, pp. 8191, 1979.

[5] H. A. Ali, M. Lippmann, U. Mundathaje, and G. Khaleeq, "Spontaneous hemothorax: a comprehensive review," Chest, vol. 134, no. 5, pp. 1056-1065, 2008.

[6] Y. Ootaki, M. Okada, C. Yamashita, T. Sugimoto, and H. Wakiyama, "Tension hemothorax caused by a ruptured aneurysm of the descending thoracic aorta: report of a case," Surgery Today, vol. 30, no. 6, pp. 558-560, 2000.

[7] K. Akiyama, A. Takazawa, J. Hirota, H. Yamagishi, and T. Akazawa, "A case of ruptured descending thoracic aortic aneurysm into the right pleural cavity: importance of preoperative drainage of the right pleural cavity," Journal of Cardiovascular Surgery, vol. 39, no. 6, pp. 869-871, 1998.

[8] J. Kokotsakis, I. Kaskarelis, P. Misthos et al., "Endovascular versus open repair for blunt thoracic aortic injury: short-term results," Annals of Thoracic Surgery, vol. 84, no. 6, pp. 19651970, 2007.

[9] N. D. Desai, A. Pochettino, W. Y. Szeto, G. W. Moser, P. J. Moeller, N. Sodhi et al., "Thoracic endovascular aortic repair: evolution of therapy, patterns of use, and results in a 10-year experience," The Journal of Thoracic and Cardiovascular Surgery, vol. 142, pp. 587-594, 2011. 


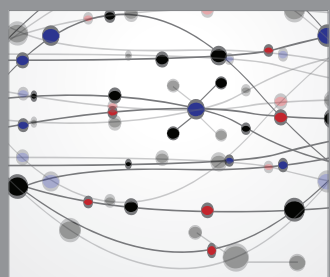

The Scientific World Journal
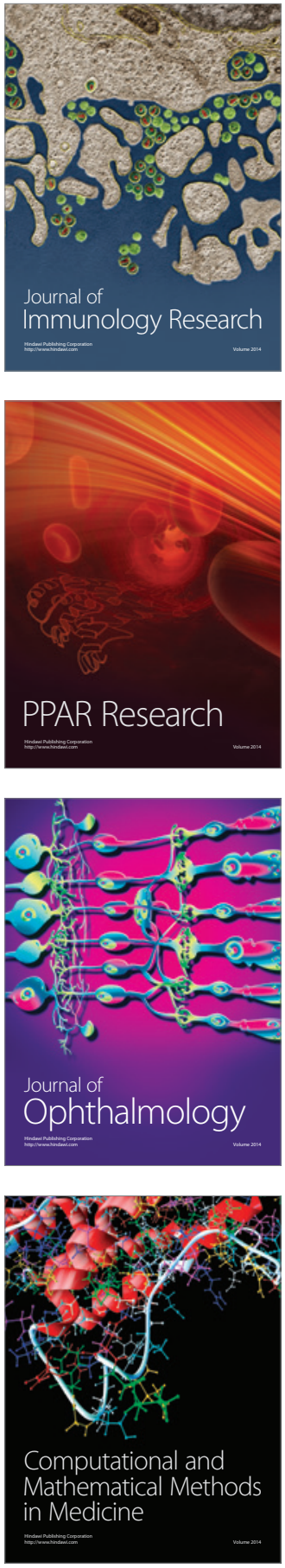

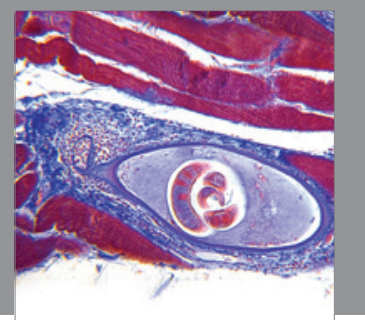

Gastroenterology

Research and Practice
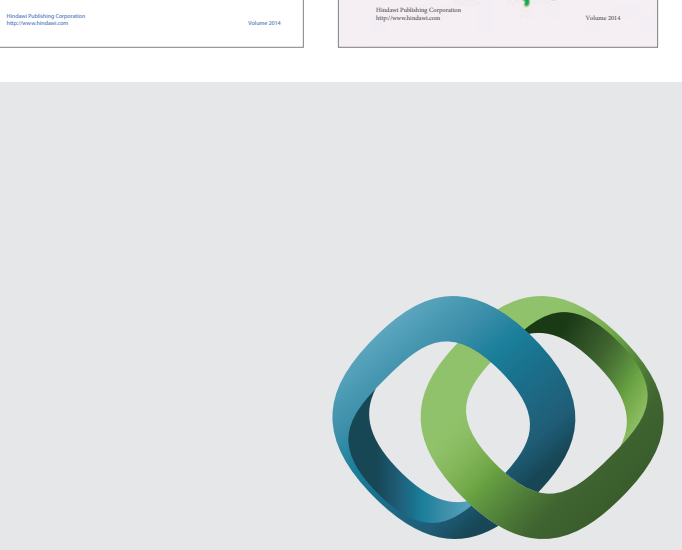

\section{Hindawi}

Submit your manuscripts at

http://www.hindawi.com
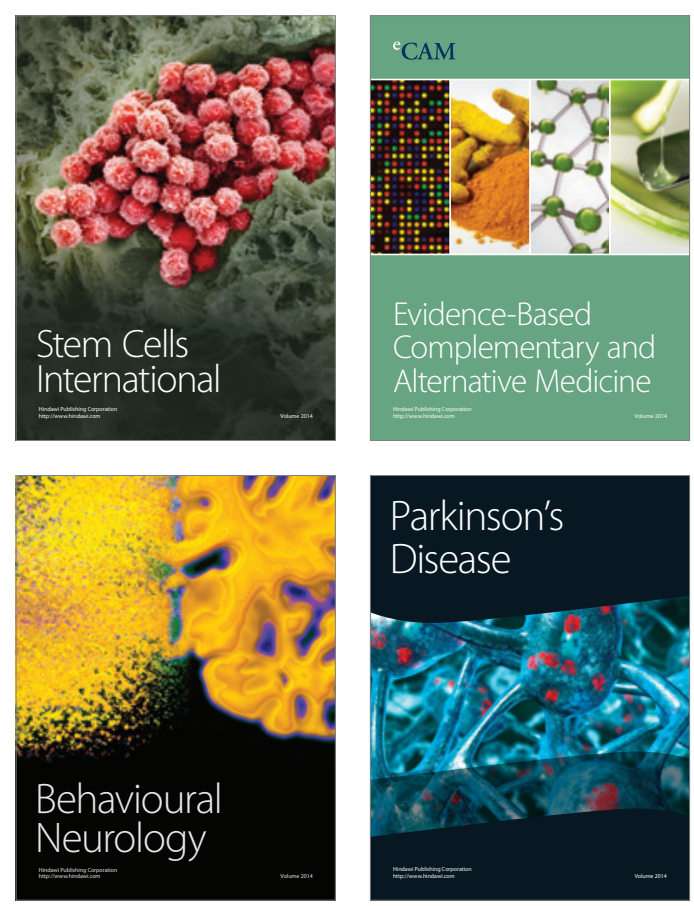

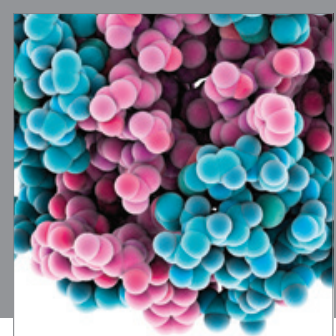

Journal of
Diabetes Research

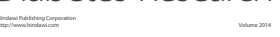

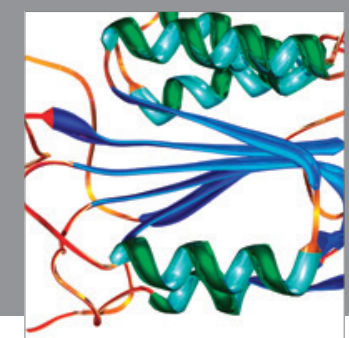

Disease Markers
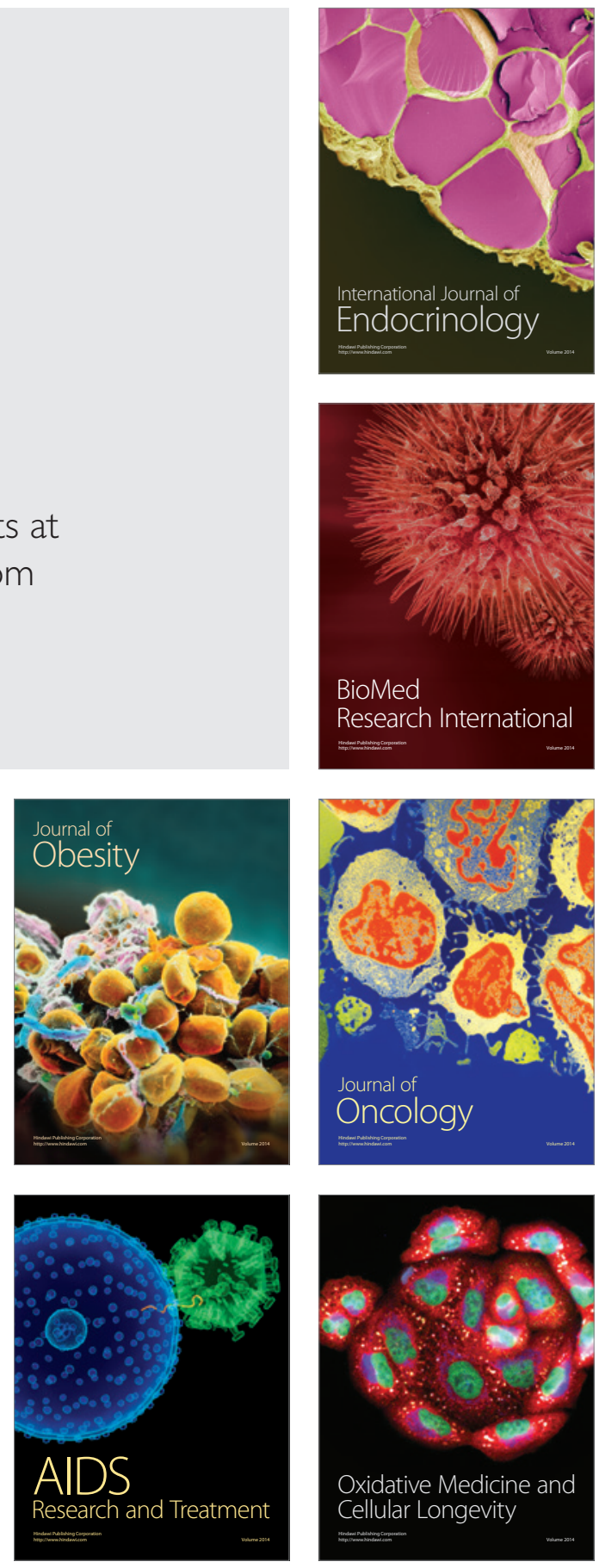